\title{
BMJ Open Recurrent acute pancreatitis prevention by the elimination of alcohol and cigarette smoking (REAPPEAR): protocol of a randomised controlled trial and a cohort study
}

Klementina Ocskay (D) , ${ }^{1,2}$ Márk Félix Juhász, ${ }^{3}$ Nelli Farkas, ${ }^{3,4}$ Noémi Zádori, ${ }^{3}$ Lajos Szakó, ${ }^{3}$ Zsolt Szakács, ${ }^{1}$ Andrea Szentesi, ${ }^{1}$ Bálint Erőss, ${ }^{1,2}$ Emőke Miklós, ${ }^{3}$ Antal Zemplényi, ${ }^{5,6}$ Béla Birkás, ${ }^{7}$ Árpád Csathó, ${ }^{7}$ István Hartung, ${ }^{7}$ Tamás Nagy, ${ }^{8}$ László Czopf, ${ }^{9}$ Ferenc Izbéki, ${ }^{10}$ László Gajdán, ${ }^{10}$ Mária Papp, ${ }^{11}$ László Czakó (D),${ }^{12}$ Dóra Illés (D) , ${ }^{12}$ Marco V Marino, ${ }^{13}$ Antonello Mirabella, ${ }^{13}$ Ewa Małecka-Panas, ${ }^{14}$ Hubert Zatorski, ${ }^{14}$ Yaroslav Susak, ${ }^{15}$ Kristina Opalchuk, ${ }^{15}$ Gabriele Capurso, ${ }^{16}$ Laura Apadula, ${ }^{16}$ Cristian Gheorghe, ${ }^{17,18}$ Ionut Adrian Saizu, ${ }^{17,18}$ Ole H Petersen, ${ }^{19}$ Enrique de-Madaria, ${ }^{20}$ Jonas Rosendahl, ${ }^{21}$ Andrea Párniczky, ${ }^{3,22}$ Péter Hegyi (D) ,,2 On behalf of the Hungarian Pancreatic Study Group

To cite: Ocskay K, Juhász MF, Farkas N, et al. Recurrent acute pancreatitis prevention by the elimination of alcohol and cigarette smoking (REAPPEAR): protocol of a randomised controlled trial and a cohort study. BMJ Open 2022;12:e050821. doi:10.1136/ bmjopen-2021-050821

- Prepublication history and additional supplemental material for this paper are available online. To view these files, please visit the journal online (http://dx.doi.org/10.1136/ bmjopen-2021-050821)

$\mathrm{AP}$ and $\mathrm{PH}$ contributed equally.

Received 01 March 2021 Accepted 12 November 2021

Check for updates

(c) Author(s) (or their employer(s)) 2022. Re-use permitted under CC BY-NC. No commercial re-use. See rights and permissions. Published by BMJ.

For numbered affiliations see end of article.

Correspondence to Professor Péter Hegyi; hegyi2009@gmail.com

\section{ABSTRACT}

Background/objectives Acute recurrent pancreatitis (ARP) due to alcohol and/or tobacco abuse is a preventable disease which lowers quality of life and can lead to chronic pancreatitis. The REAPPEAR study aims to investigate whether a combined patient education and cessation programme for smoking and alcohol prevents ARP. Methods and analysis The REAPPEAR study consists of an international multicentre randomised controlled trial (REAPPEAR-T) testing the efficacy of a cessation programme on alcohol and smoking and a prospective cohort study (REAPPEAR-C) assessing the effects of change in alcohol consumption and smoking (irrespective of intervention). Daily smoker patients hospitalised with alcohol-induced acute pancreatitis (AP) will be enrolled. All patients will receive a standard intervention priorly to encourage alcohol and smoking cessation. Participants will be subjected to laboratory testing, measurement of blood pressure and body mass index and will provide blood, hair and urine samples for later biomarker analysis. Addiction, motivation to change, socioeconomic status and quality of life will be evaluated with questionnaires. In the trial, patients will be randomised either to the cessation programme with 3-monthly visits or to the control group with annual visits. Participants of the cessation programme will receive a brief intervention at every visit with direct feedback on their alcohol consumption based on laboratory results. The primary endpoint will be the composite of 2-year all-cause recurrence rate of AP and/ or 2-year all-cause mortality. The cost-effectiveness of the cessation programme will be evaluated. An estimated 182 participants will be enrolled per group to the REAPPEAR-T with further enrolment to the cohort.

Ethics and dissemination The study was approved by the Scientific and Research Ethics Committee of the

\section{Strengths and limitations of this study}

- This is the first study assessing a combined brief intervention programme for recurrence prevention in acute pancreatitis.

- The study could provide a cost-effective and easyto-use preventive method, reducing the recurrence rate of alcoholic acute pancreatitis.

- The lack of a conventional control group could result in underestimating the efficacy of the cessation programme.

- The results will be specific to the enrolled patient population, which does not cover all patients with recurrent acute pancreatitis.

Hungarian Medical Research Council (40394-10/2020/ EÜIG), all local ethical approvals are in place. Results will be disseminated at conferences and in peer-reviewed journals.

Trial registration number NCT04647097

\section{INTRODUCTION}

Acute pancreatitis (AP) is an often-unheeded issue by clinicians and healthcare professionals, with significant medical charges. ${ }^{12}$ The incidence rate of the first attack of AP ranges from 15 to 45 per 100000 per year. $^{3}$ Alcohol and biliary obstruction are the two main causes of AP in adulthood, alcohol being the diagnosed inducing factor in $25 \%-35 \%$ of the cases. ${ }^{4}$ 
Cohort studies have found that $10 \%-30 \%$ of patients have recurrent attacks based on medical history, and a recent meta-analysis has shown that $10 \%$ of the patients after a single episode of AP and $26 \%$ of those with acute recurrent pancreatitis (ARP) later progress to chronic pancreatitis (CP) ${ }^{5}$ It Is known, that ARP (more than one episodes of AP) significantly lowers physical and mental quality of life $(\mathrm{QoL})^{6}$ and alcoholic aetiology has been identified in $19 \%$ of ARP patients. ${ }^{7}$ Despite the importance and potentially preventable nature of alcoholic ARP, preventive efforts are still scarce. ${ }^{89}$

A pivotal study from Nikkola et al found that abstinent patients experienced no ARPs during a 9-year follow-up period. On the other hand, $34 \%$ of patients who did not stop drinking developed a recurrent attack. ${ }^{10}$ The median time between the index AP and the first alcoholic ARP ranges from 8.5 months to 2.2 years, but around $80 \%$ of the registered first recurrent attacks occur in the first 4 years of follow-up. ${ }^{11}{ }^{12}$ With 6-monthly interventions, Nordback et al achieved a significant reduction in the recurrence rate of AP in Finland. ${ }^{13} 14$

Smoking is a long-established independent risk factor of AP and CP. A dose-response association was found between smoking and $\mathrm{AP},{ }^{15}{ }^{16}$ and combined with heavy drinking, smoking can further increase the risk of AP up to four times compared with non-smokers. ${ }^{17}{ }^{18}$ Findings are controversial regarding the effects of smoking cessation. A study published by Sadr-Azodi found that the risk of AP is statistically comparable to never-smokers' after 20 non-smoking years. ${ }^{17}$ In contrast, a meta-analysis showed an elevated risk of AP in former smokers compared with never-smokers. ${ }^{18}$

Limiting alcohol use and smoking apart from their positive effects on the pancreas generally improve health ${ }^{19}$ and up to a certain extent, organ damage caused by these substances is reversible. ${ }^{20-23}$ Smoking cessation alone can prolong life with $1.4-8.5$ years. $^{24}$

In a Hungarian cohort study of 600 patients, alcohol consumption was four times more frequent in males, alcoholic aetiology represented $26.5 \%$ of all cases and was often associated with smoking. Alcoholic ARP accounted for $21.2 \%$ of all cases in the cohort. ${ }^{25}$ In a CP cohort, daily alcohol consumption, as an etiological factor, was present in $56 \%$ of the cases, and $56 \%$ of the participants smoked more than 10 cigarettes/day. ${ }^{26}$

It is known that more than half of patients suffering from alcohol use disorder (AUD) are also dependent on tobacco, and that continued tobacco use represents a more than two-fold risk for relapse. ${ }^{27} 28$ To this day, there are no adjusted protocols for the treatment and follow-up of heavy-drinking smokers. ${ }^{29}{ }^{30}$ It is proven that, in contrast with previous assumptions, smoking cessation programmes for patients at risk or living with AUD improve alcohol-related outcomes ${ }^{2731}$ and a brief alcohol intervention improves the rate of successful smoking cessation. ${ }^{32}$

However, to date, no study has examined the effects of a combined intervention for the reduction of nicotine and alcohol consumption in ARP and guidance is very limited on this topic, ${ }^{33-36}$ (online supplemental table S1). Based on the above-mentioned reasons, while all patients with alcoholic AP should receive counselling, a one-time brief intervention will be provided to all participants, without further counselling in the control group.

\section{Objectives}

The study encompasses a randomised controlled trial (RCT) (REAPPEAR-T: Recurrent acute pancreatitis prevention by the elimination of alcohol and cigarette smoking; trial) and a concomitant cohort study (REAPPEAR-C: Recurrent acute pancreatitis prevention by the elimination of alcohol and cigarette smoking; cohort). The REAPPEAR-T's objective is to investigate the effect of an alcohol and smoking cessation programme combined with patient education on the recurrence rate of alcoholinduced AP, CP and QoL. Additionally, the REAPPEAR-C's objective is to investigate the effect of alcohol and smoking cessation (irrespective of intervention) on the recurrence rate of alcohol-induced AP, CP and QoL.

\section{METHODS}

\section{Design}

The REAPPEAR study, designed in accordance with the Standard Protocol Items: Recommendations for Interventional Trials (SPIRIT) statement, ${ }^{37}$ uses a combined design to answer two questions in one particular patient population. The REAPPEAR-T will be an international, single-blind, two-arm, parallel group, superiority RCT, testing the efficacy of a cessation programme for alcohol and smoking, using brief interventions. The REAPPEAR-C is a prospective four-arm cohort study, which includes all patients participating in REAPPEAR-T with further enrolment after the termination of enrolment to the trial. In the cohort, patients will be grouped by smoking status and alcohol consumption at the end of the study, irrespective of intervention. The same eligibility criteria and outcomes will be used in both substudies and differences will be described in the appropriate sections in detail.

The study will be conducted in Hungary, Ukraine, Italy and Romania (list of centres in online supplemental file $1)$. Centres are welcome to join. The enhance the visibility of this project and centre recruitment, the protocol is being presented on national and international conferences. Patients will be enrolled during their hospitalisation for AP and will be followed during ambulatory visits to the same hospital.

\section{Population}

\section{Inclusion criteria}

- Patient hospitalised with alcohol-induced AP (defined by the revised Atlanta criteria. ${ }^{38}$

- Regular consumption of at least $40 \mathrm{~g}$ (women)/ $50 \mathrm{~g}$ (men) alcohol daily or $280 \mathrm{~g}$ (women) / $350 \mathrm{~g}$ (men) alcohol during the preceding week of onset of abdominal pain 
- Every day smoker (defined as an adult patient who smoked at least 100 cigarettes in his or her lifetime, and now smokes on a daily basis; as per the CDC definition), with at least 1-year history of smoking.

- Aged 18-65 years. ${ }^{39}$

- Completed the standard intervention (SI) (see below).

- Provided written informed consent (online supplemental file 2).

\section{Exclusion criteria}

- Possible aetiologies for AP other than alcohol (eg, gallstone-related, hypertriglyceridaemia above 11.5 $\mathrm{mM},{ }^{40-42}$ hypercalcaemia, viral infection) and cases with combined etiological factors will be excluded.

- Major psychiatric illnesses (eg, schizophrenia, bipolar disorder, dementia).

- Currently receiving therapy for AUD.

- Currently taking part in a smoking cessation programme.

- Three or more documented lifetime episodes of AP. ${ }^{43}$

- $\mathrm{CP}^{44}$

- Undergoing active or palliative treatment for malignancy.

- Pregnancy.

- Life expectancy is less than 2 years.

Medical personnel not involved in the treatment of the patient will perform formal screening and obtain informed consent.

\section{Standard intervention}

The SI will be incorporated into standard medical therapy in all centres, and will be provided to all patients hospitalised for alcohol-induced AP. SI will be delivered by a specially trained nurse because interventions delivered by nurses have been found to be the most effective in reducing the quantity of alcohol consumed. ${ }^{45}$ The intervention will be based on the WHO initiative 'Assistlinked brief intervention', using psychoeducational and motivational interviewing techniques. ${ }^{46}$ For SI, we calculated with an average length of $30 \mathrm{~min}$, based on a recent Cochrane review including 69 RCTs, according to which longer interventions on alcohol had no benefit, the median duration being $25 \mathrm{~min} .{ }^{47}$ SI will also provide educational information about the nature of alcoholic AP and the risk of recurrence to the patients. Feasibility and cost-effectiveness were also considered.

\section{Intervention in REAPPEAR-T}

The repeated intervention will be provided by the same specially trained personnel and structured similarly to the SI. Each session will have the same structure but can be tailored to the patient's needs to strengthen motivation. Sessions will consist of three parts. First, the negative effects of alcohol and smoking on the pancreas will be highlighted. Second, the patient's motivation for abstinence and smoking cessation will be discussed. Third, the individual's responsibility in achieving the goals set after motivation assessment, with personalised advice. ${ }^{13}$ We wish to enhance the efficacy of the repeated intervention by providing feedback for the patient based on the mean corpuscular volume and gamma glutamyl-transferase (GGT) values measured at the day of the interview. ${ }^{48}$ The trained personnel providing the interventions will not take part in patient care in any form. A detailed protocol will be provided on request.

\section{Concomitant care}

Patients participating in cessation programmes or psychotherapy at the time of enrolment will not be eligible. Patients using self-help programmes and nicotine replacement therapy with commonly available products will not be excluded. The provided interventions encourage patients to seek help and try different strategies for alcohol and smoking cessation.

\section{Outcomes}

Primary endpoint

The primary endpoint of the REAPPEAR Study will be the composite of 2-year recurrence rate of AP irrespective of aetiology and 2-year all-cause mortality.

\section{Secondary endpoints}

1. ARP irrespective of aetiology (given as cumulative incidence and as rate of event) within 6, 12, 18 and 24 months.

2. Recurrence of alcohol-induced AP (rate of event) within 2 years.

3. The condition of 'likely pancreatitis' (fulfilling the diagnostic criteria of epigastric pain, a serum amylase or lipase level at least two times the upper normal level, and elevated leucocyte count or CRP levels, defined by Pelli et al. ${ }^{49}$

4. Length of hospital stay given in days (specifically due to recurrent pancreatitis and cumulative during followup).

5. Presentation to the emergency unit with and without hospital readmission (cumulative incidence).

6. Change of alcohol consumption and tobacco use (compared with baseline), estimated separately form biomarker levels and patient-reported consumption

7. CP (incidence within 2 years). ${ }^{44}$

8. Changes in body mass index (BMI) and blood pressure (compared with baseline).

9. Healthcare cost from the perspective of the health insurance fund within 2 years and quality-adjusted lifeyears (QALY).

\section{Recruitment}

Consecutively, all patients under treatment for alcoholinduced AP who received the SI according to standard protocol will be screened for eligibility, all eligible patients will be offered to participate in the REAPPEAR study. The potential benefits of participation will be highlighted to facilitate patient recruitment. The planned start and end dates of patient recruitment are 1 March 2021 and 1 December 2024. 


\section{Biologic sample collection and biomarker measurements}

At enrolment and every visit, basic laboratory tests from blood will be carried out and participants will provide blood, hair and urine samples for storage in the biobank.

Laboratory parameters measured are shown in online supplemental file 3. Laboratory results will be evaluated by a physician, who will decide whether further medical attention is necessary. All patients will receive the results of their laboratory tests in written form.

The samples in the biobank will be stored at $-80^{\circ} \mathrm{C}$ and identified by the personal identification number (PIN) given at study entry. Planned alcohol and smoking biomarker measurements include urine and serum ethylglucuronide (or ethyl-sulfate) and hair nicotine measurements. ${ }^{50}$ All samples will be collected and sent together to the laboratory when the patient number reached the preset goal for analysis. The results of the biomarker measurements will not be made accessible for patients. These measurements are only available in specialised laboratories, therefore, can be changed later due to feasibility issues.

\section{Trial organisation, committees and boards}

The corresponding centre of the REAPPEAR study is the Centre for Translational Medicine, Medical School, University of Pécs (www.tm-centre.org), whereas the coordinator and designer research team is the Hungarian Pancreatic Study Group (HPSG, https://tm-centre.org/ en/study-groups/hungarian-pancreatic-study-group/). HPSG has been running high-quality international, multicentre clinical trials since $2014^{4152-54}$ and has published relevant guidelines for pancreatic diseases to improve patient care in pancreatology. ${ }^{556}$

The steering committee (SC) will be led by PH (principal investigator, specialist in internal medicine, gastroenterology and clinical pharmacology). SC members will be KO (study coordinator), a patient representative, NF (biostatistician), IH (psychologist) and the centre leaders. The SC will supervise the trial primarily and will make decisions regarding all critical questions overseeing patient safety, the progress of the trial, adherence to protocol, considering new information relevant to the trial and ensuring dissemination and implementation of the results.

All data gathered for research purposes will be handled confidentially and anonymously, which will be ensured by the data monitoring committee (DMC). Six-monthly audits are planned in each centre with continuous monitoring of the electronic case report forms (eCRFs) (online supplemental file 3) For each participant, a PIN will be generated and it will be present on all forms and documents of each individual.

The International Advisory Board will include Ole Petersen, Enrique de-Madaria and Jonas Rosendahl, providing independent external advice and guidance on strategic matters.

The study was designed by the SC and was supported by the University of Pécs Medical School. The sponsor had no role in the design of the trial and will have no access to the randomisation codes or the data. The sponsor will not participate in data monitoring, analysis and publication of results.

The independent safety monitor will be LC, who will ensure the safety of the patients and revise all reported harms possibly related to the intervention.

\section{Data handling}

Investigators will be responsible for the accuracy, reliability and quality of the collected data. Detailed data flow will be described in a data management plan. Data from completed eCRFs will be validated under the direction of the data manager on the DMC according to the data cleaning plan. Any missing, implausible or inconsistent recordings in the eCRFs will be referred back to the Investigator using a data query form and will be documented for each subject before clean file status is declared. All changes to eCRFs will be recorded.

The DMC will perform an independent assessment of trial-related documents and activities to ensure respect for subjects' rights, safety and well-being and to guarantee the plausibility of clinical data. The similarity of groups at baseline will also be checked.

After written consent of the subjects, data will be recorded by the investigators. Clinical research data will be processed separately from participants' personal data. Data may only be accessed by a person acting under the authority of the controller and in accordance with the authorisation system established within the controller's organisational structure, only to the extent and in the manner necessary for the performance of tasks. Personal data will not be made accessible to third parties. We will fully comply with the General Data Protection Regulation (GDPR).

\section{Safety}

Based on the nature of the combined brief intervention in REAPPEAR-T, we do not expect serious adverse events. However, minor or moderate adverse events may occur. Participants will be provided with information on alcohol and nicotine withdrawal alongside with the available options of professional help for addiction treatment. In case a potentially serious health problem is detected by the investigators related to the intervention, the safety monitoring board will be notified. The REAPPEAR-C is an observational study, hence adverse events are not applicable.

\section{Randomisation and allocation concealment in REAPPEAR-T}

Central randomisation will be performed with randomly permuted block size (2-6) and allocation ratio of 1:1 using a computer-generated random sequence. Inclusion and exclusion criteria will be rechecked prior to computer-aided randomisation via an online platform. The platform generates the PIN and a follow-up plan (with appointment dates). The randomisation procedure will be performed by the same person who screened and 
consented the patient. This person must be a doctor not actively participating in the treatment of the participant.

\section{Blinding in REAPPEAR-T}

Outcome assessors will be blinded to allocation. The medical personnel involved in the check-ups and treatment during a potential hospital re-admission will not be aware of the allocation. Since the nature of the intervention, the patient and the study nurse cannot be properly blinded.

\section{Statistical analysis}

Sample size calculation for REAPPEAR-T was based on the only published interventional randomised study assessing the effects of repeated brief interventions in alcohol-induced ARP, counting with a 2-year recurrence rate of $21.3 \%$ and an absolute reduction to $8.5 \% .^{13151725}$ Considering one interim analysis on efficacy (with the Pocock correction), $80 \%$ power, $5 \%$ alpha (superiority design, two-sided) and a drop-out rate of $30 \%$, the estimated sample size is 182 subject per study arm. This sample size calculation is expected to overestimate the minimum number of participants for three reasons: (1) the use of a combined intervention on alcohol and smoking and more frequent visits are expected to result in greater reduction of recurrence, (2) the use of a composite primary endpoint may result in higher event numbers and (3) the recurrence rate of AP is expected to be higher in the heavy-drinking smoker population, than in a mixed sample. The calculation was performed by Stata (V.15).

Safety analysis will be carried out on reaching $10 \%$ of the target patient enrolment, and a single interim analysis for efficacy and sample size re-estimation taking into consideration the observed drop-out rate at $50 \%$ of the expected total events of the primary outcome, which is 21. Early stopping will be executed (1) if safety concerns arise during the interim analysis or anytime later (stopping for safety concerns), (2) if the statistical power reaches at least $80 \%$ and $\mathrm{p}<0.05$ for the primary outcome at the interim analysis (stopping for benefit), (3) if the results of the interim analysis show equal effects in both groups (stopping for futility) and (4) if power does not reach $80 \%$, sample size will be re-estimated using the observed event and drop-out rate. In case the newly calculated sample size is unfeasible for the trial, both groups will continue follow-up according to the schedule of REAPPEAR-C (stopping for feasibility).

In the final analysis, intention-to-treat will be favoured over per-protocol (or 'as-treated') analysis. Information on mortality and hospitalisations will be obtained from the organisation responsible for handling data.

The 'last observation carried forward' strategy will be followed to impute missing data for other outcomes measured during the study.

Sample size calculation for the REAPPEAR-C will be carried out at the final analysis of the REAPPEAR-T, using available data from participants. Further enrolment will be performed according to the estimated sample size. These additional participants will receive the more effective or in case of equality the less costly intervention for alcohol and smoking cessation as determined by the results of the REAPPEAR-T. Participants of the cohort will be categorised into four groups primarily, according to smoking and drinking status (quit smoking; quit drinking; quit both; still smokes and drinks). Time of smoking and alcohol cessation will be taken into consideration. Participants who started smoking or drinking again after an abstinent period will be excluded from analysis in the REAPPEAR-C.

In descriptive statistics, the count and percentage will be provided for each treatment arm for binary outcomes. For continuous outcomes, number (n), mean, median, IQR (Q3-Q1), SD, minimum and maximum values will be provided for each treatment arm. In the univariate comparative analysis, we will calculate relative risk with 95\% CI when comparing the primary endpoint between two groups (alpha $=5 \%$ ) with a reference arm using the control group complemented with $\chi^{2}$ or Fisher's exact test (the same strategy will be followed for binary secondary outcomes). For continuous variables, we will use t-test assuming unequal variances or the Mann-Whitney test. We will perform univariate (Kaplan-Meier and Cox regression) and multivariate (Cox regression) survival analyses for binary outcomes. An adjustment will be carried out at least for age, sex, socioeconomic status, the number of prior ARPs, comorbidities, history of alcohol consumption (cumulative) and smoking (package year), severity and complications of index AP, BMI, cholecystectomy and enrolling centre. Mixed effect logistic regression will be conducted to estimate the effect of the multicomponent intervention on the outcomes, where the subject PINs will be used as a random subject. The model will be adjusted for changes in smoking habits, alcohol consumption, BMI, socioeconomic status, blood pressure and Maddrey score. $^{57}$

All analyses will be carried out with SPSS V.26 and Stata V.15.

\section{Drop-outs}

Information on the primary outcome will be obtained either from the patient's documentation or from the National Health Insurance Fund or similar organisation managing data on healthcare costs and mortality, therefore information on the primary outcome will be available for most patients regardless of attendance of the study visits. Only withdrawal of consent will result in missing data.

Considering per-protocol analysis, in the REAPPEAR-T trial, missing more than one consecutive interventions after the initial assessment or withdrawal of consent during follow-up will result in the drop-out of the patient. In the REAPPEAR-C investigation, patients who withdraw consent during follow-up or miss the 2-year visit will be considered drop-outs, since data on current alcohol 


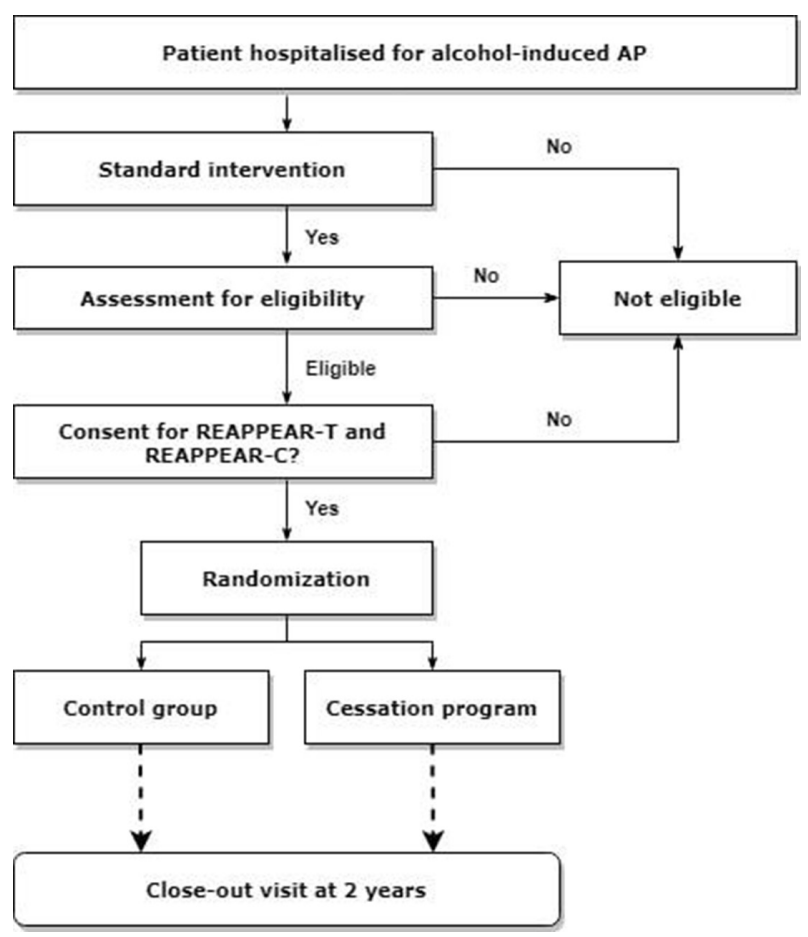

Figure 1 SPIRIT flow chart. Standard intervention will be provided for every patient as part of standard therapy. All randomised participants will be included in the REAPPEAR-T (trial) and REAPPEAR-C (cohort) as well. After reaching the required patient numbers for the REAPPEAR-T, further patients will be enrolled to the REAPPEAR-C in accordance with the estimated sample size. AP, acute pancreatitis. SPIRIT, Standard Protocol Items: Recommendations for Interventional Trials, REAPPEAR, Recurrent acute pancreatitis prevention by the elimination of alcohol and cigarette smoking.

consumption and smoking can only be obtained from the patient.

\section{Flow and timing}

Patients who met the inclusion criteria and none of the exclusion criteria will be offered to participate in the REAPPER-T trial. The enrolment period lasts from 48 hours before, until 1 week after hospital discharge. After informed consent and randomisation, participants will be assigned to the cessation programme or the control group (see at figure 1). All patients will appear at the clinic

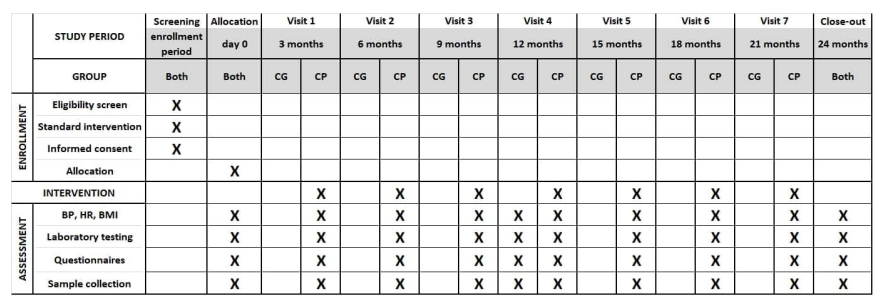

Figure 2 SPIRIT time table. SPIRIT, Standard Protocol Items: Recommendations for Interventional Trials; BMI, body mass index; BP, blood pressure; CG; control group, CP; cessation programme, HR; heart rate. according to the study schedule (figure 2), within \pm 14 days from the prescheduled date.

We chose 3-monthly visits in the cessation programme based on a Swedish cohort study, in which 3-monthly brief interventions for selected patients with increased GGT levels were introduced and GGT levels were used for feedback. These interventions were found to reduce mortality, hospitalisation and sick leave significantly. ${ }^{48}$ Hopefully, frequent visits will help in upholding motivation and improve adherence. Patients in the control group will have two prescheduled appointments, at 12 and 24 months.

\section{Assessment}

For the assessment of addiction and motivation to quit will be assessed by internationally recognised and validated questionnaires (online supplemental file 3). ${ }^{58-64}$ This will enable the person who provides the intervention to individualise it and motivate the subject. Data on coffee consumption will be collected as well, as caffeine might counter the effects of alcohol in AP. ${ }^{65}$ For the assessment of QoL, the EQ-5D-5L questionnaire will be used at baseline and every visit. ${ }^{66}{ }^{67}$ Socioeconomic status will be assessed at baseline and at 12 and 24 months with the questionnaire used in the LIFESPAN study. ${ }^{68}$

The aetiology of each recurrent episode will be determined following current international guidelines, but all episodes will be included in the primary endpoint. ${ }^{434}$

Blood pressure, heart rate and body weight will be measured by an independent nurse blind to the allocation at every visit. BMI will be calculated.

\section{Cost-effectiveness}

Cost-effectiveness analysis will be performed to examine the impact of the cessation programme on QoL, survival and health expenditure compared with the controls. We calculate the incremental cost-effectiveness ratio (ICER), which is defined by the difference in cost between the compared interventions (cessation programme with 3-monthly visits vs usual care), divided by the difference in their effect (QALY). The ICER will be evaluated based on the Hungarian cost-effectiveness threshold. The total cost of treatment per each individual will be obtained from the national database at the completion of the study.

\section{Patient and public involvement}

Five randomly selected patients from the HPSG database were invited. All of them had previous AP and would have been eligible for the study. Three patients attended the joint consultation. The original aims, hypotheses and protocol of the study were fully introduced to them. Patients insights were as follows: (1) they welcomed the study with great pleasure and felt it is highly important, (2) they found the primary endpoint fundamental, (3) they found the questionnaires and information sheets understandable, (4) they highlighted the importance of frequent visits to the clinic, and found the duration of the visits feasible, (5) they pointed out the necessity of 
high quality training of personnel providing the interventions, (6) they had absolutely no disapproval or negative feelings regarding regular blood tests, (7) they had no ethical objection concerning the control group and (8) they expressed high difficulties considering smoking cessation and favoured a step-down approach rather than immediate quitting.

We have revised and modified the original protocol accordingly.

\section{DISCUSSION}

Although alcohol and smoking are individual risk factors for AP, ARP and CP, they can synergise each other's effects. ${ }^{69}$ In addition, there is a lack of evidence as to the means of preventive measures that could be used in everyday clinical practice concerning alcohol and tobacco use for AP patients. Also, the effect of smoking cessation on recurrence in drinkers and non-drinkers is not yet clear.

The REAPPEAR study aims to fill these gaps and provide specialists and primary care physicians with valuable information on the importance of alcohol and smoking cessation in AP and ARP. Furthermore, the feasibility, efficacy and cost-effectiveness of an intervention programme will be tested in this population to provide basis for largescale intervention in alcohol-induced pancreatitis.

\section{ETHICS AND DISSEMINATION}

The REAPPEAR study is open for participation. Results of the planned analyses will be presented at national and international conferences and in peer-reviewed journals. Additional long-term follow-up of the participants is planned within the confines of the REAPPEAR+study.

The trial has been registered at the clinicaltrials.gov (NCT04647097). Amendments will be published under this registration number.

The Scientific and Research Ethics Committee of the Hungarian Medical Research Council approved the study (40394-10/2020/EÜIG). All local ethical approvals are in place. The study will be performed in accordance with the declaration of Helsinki, the principles of International Conference on Harmonisation-Good Clinical Practice (ICH-GCP) guidelines and local legal and regulatory requirements.

\footnotetext{
Author affiliations

${ }^{1}$ Institute for Translational Medicine, Szentágothai Research Centre, Medical School, University of Pécs, Pécs, Hungary

${ }^{2}$ Centre for Translational Medicine, Semmelweis University, Budapest, Hungary ${ }^{3}$ Institute for Translational Medicine, Medical School, University of Pécs, Pécs, Hungary

${ }^{4}$ Medical School, Institute of Bioanalysis, University of Pécs, Pécs, Hungary ${ }^{5}$ Faculty of Pharmacy, Division of Pharmacoeconomics, University of Pécs, Pécs, Hungary

${ }^{6}$ Center for Health Technology Assessment, University of Pécs, Pécs, Hungary ${ }^{7}$ Medical School, Institute of Behavioural Sciences, University of Pécs, Pécs, Hungary
}

${ }^{8}$ Medical School, Department of Laboratory Medicine, University of Pécs, Pécs, Hungary

${ }^{9}$ Medical School, First Department of Medicine, Division of Cardiology and Angiology, University of Pécs, Pécs, Hungary

${ }^{10}$ First Department of Internal Medicine, Szent György University Teaching Hospital of Fejér County, Székesfehérvár, Hungary

${ }^{11}$ Department of Internal Medicine, Division of Gastroenterology, University of Debrecen, Debrecen, Hungary

${ }^{12}$ Faculty of Medicine, First Department of Medicine, University of Szeged, Szeged, Hungary

${ }^{13}$ General and Emergency Surgery Department, Azienda Ospedaliera Ospedali Riuniti Villa Sofia-Cervello, Palermo, Italy

${ }^{14}$ Department of Digestive Tract Diseases, Medical University of Lodz, Lodz, Poland

${ }^{15}$ Department of Surgery, Bogomolets National Medical University, Kyiv, Ukraine

${ }^{16}$ Pancreas Translational and Clinical Research Center, San Raffaele Scientific Institute, Vita Salute San Raffaele University, Milan, Italy

${ }^{17}$ Clinical Institute Fundeni, Bucuresti, Romania

${ }^{18} \mathrm{Carol}$ Davila University of Medicine and Pharmacy, Bucuresti, Romania

${ }^{19}$ Cardiff University, Cardiff, UK

${ }^{20}$ Gastroenterology Department, Alicante University General Hospital, Alicante Institute for Health and Biomedical Research (ISABIAL), Alicante, Spain

${ }^{21}$ Department of Internal Medicine I, Martin Luther University, Halle, Germany

${ }^{22}$ Heim Pál National Pediatric Institute, Budapest, Hungary

Twitter lonut Adrian Saizu @saizuadrian and Enrique de-Madaria @DeMadaria

Acknowledgements We would like to thank the interdisciplinary team of the Institute for Translational Medicine and the staff from all centres.

Contributors All authors were involved in the study design and edited the manuscript. KOc conducted the literature search, conceptualised and wrote the manuscript and prepared the figures. FJ participated in manuscript writing, critical revision, abstract writing and figure preparation. NF performed the sample size calculation and planned the statistical analyses. NZ and LS facilitated the ethical approval and trial registration. ZS provided methodological guidance, critically reviewed and revised the manuscript, EM facilitated patient invovlement and will coordinate data management, $\mathrm{BB}, \mathrm{AC}$ and $\mathrm{IH}$ designed the intervention and chose the questionnaires, AZ will carry out the healthcare cost calculations. LC provided expert opinion and will perform safety monitoring. TN provided expert opinion on laboratory measurements and biological sample collection. FI, LG, MP, LC, DI, MVM, AM, EM-P, HZ, YS, KOp, GC, LA, CG and IAS provided insight on feasibility and will coordinate local enrolment and follow-up. OHP, Ed-M and JR provided expert opinion. $\mathrm{PH}, \mathrm{AP}, \mathrm{AS}, \mathrm{BE}$ critically reviewed and revised the manuscript. $\mathrm{PH}$ and $\mathrm{AP}$ have given significant intellectual input and supervised the work. All authors have read and approved the final version of the manuscript. AP and PH co-supervised this work as joint last authors.

Funding Center costs (IT, biostatistics, trial organisation, etc) are covered by the University of Pécs Medical School (grant number: not applicable). This work was supported by 'GINOP-2.3.2-15-2016-00048 - STAY ALIVE' (PH) cofinanced by the European Union (European Regional Development Fund) within the framework of Programme Széchenyi 2020, and by EFOP 3.6.2-16-2017-00006 - LIVE LONGER (PH) Human Resources Development Operational Programme Grant which is cofinanced by the European Union (European Regional Development Fund) within the framework of Programme Széchenyi 2020 as well as the Translational Medicine Foundation. Further grants: a János Bolyai Research Scholarship from the Hungarian Academy of Sciences (AP) and National Research, Development and Innovation Office Project Grants (K116634, (PH);, FK138929 (AP)).

Disclaimer The sponsor was not involved in the design of the study and will have no access to the database or the randomisation code.

Competing interests None declared.

Patient consent for publication Not applicable.

Provenance and peer review Not commissioned; externally peer reviewed.

Supplemental material This content has been supplied by the author(s). It has not been vetted by BMJ Publishing Group Limited (BMJ) and may not have been peer-reviewed. Any opinions or recommendations discussed are solely those of the author(s) and are not endorsed by BMJ. BMJ disclaims all liability and responsibility arising from any reliance placed on the content. Where the content includes any translated material, BMJ does not warrant the accuracy and reliability of the translations (including but not limited to local regulations, clinical guidelines, 
terminology, drug names and drug dosages), and is not responsible for any error and/or omissions arising from translation and adaptation or otherwise.

Open access This is an open access article distributed in accordance with the Creative Commons Attribution Non Commercial (CC BY-NC 4.0) license, which permits others to distribute, remix, adapt, build upon this work non-commercially, and license their derivative works on different terms, provided the original work is properly cited, appropriate credit is given, any changes made indicated, and the use is non-commercial. See: http://creativecommons.org/licenses/by-nc/4.0/.

\section{ORCID iDs}

Klementina Ocskay http://orcid.org/0000-0001-5848-2506

László Czakó http://orcid.org/0000-0002-6331-0802

Dóra Illés http://orcid.org/0000-0003-3138-8039

Péter Hegyi http://orcid.org/0000-0003-0399-7259

\section{REFERENCES}

1 Wadhwa V, Patwardhan S, Garg SK, et al. Health care utilization and costs associated with acute pancreatitis. Pancreas 2017;46:410-5.

2 Szentesi A, Tóth E, Bálint E, et al. Analysis of research activity in gastroenterology: pancreatitis is in real danger. PLoS One;11:e0165244.

3 Machicado JD, Yadav D. Epidemiology of recurrent acute and chronic pancreatitis: similarities and differences. Dig Dis Sci 2017;62:1683-91.

4 Tenner S, Baillie J, DeWitt J, et al. American College of gastroenterology guideline: management of acute pancreatitis. Am J Gastroenterol 2013;108:1400-15

5 Guda NM, Muddana V, Whitcomb DC, et al. Recurrent acute pancreatitis: international state-of-the-science conference with recommendations. Pancreas 2018;47:653-66.

6 Coté GA, Yadav D, Abberbock JA, et al. Recurrent acute pancreatitis significantly reduces quality of life even in the absence of overt chronic pancreatitis. Am J Gastroenterol 2018;113:906-12.

7 Yadav D, O'Connell M, Papachristou Gl. Natural history following the first attack of acute pancreatitis. Am J Gastroentero 2012;107:1096-103.

8 Mandalia A, Wamsteker E-J, DiMagno MJ. Recent advances in understanding and managing acute pancreatitis. F1000Res 2018;7. doi:10.12688/f1000research.14244.1. [Epub ahead of print: 2806 2018].

9 Krishna SG, Kamboj AK, Hart PA, et al. The changing epidemiology of acute pancreatitis hospitalizations: a decade of trends and the impact of chronic pancreatitis. Pancreas 2017;46:482-8.

10 Nikkola J, Räty S, Laukkarinen J, et al. Abstinence after first acute alcohol-associated pancreatitis protects against recurrent pancreatitis and minimizes the risk of pancreatic dysfunction. Alcohol Alcohol 2013;48:483-6.

11 Magnusdottir BA, Baldursdottir MB, Kalaitzakis E, et al. Risk factors for chronic and recurrent pancreatitis after first attack of acute pancreatitis. Scand J Gastroenterol 2019;54:87-94.

12 Nikkola J, Laukkarinen J, Lahtela J, et al. The long-term prospective follow-up of pancreatic function after the first episode of acute alcoholic pancreatitis: recurrence predisposes one to pancreatic dysfunction and pancreatogenic diabetes. J Clin Gastroenterol 2017;51:183-90.

13 Nordback I, Pelli H, Lappalainen-Lehto R, et al. The recurrence of acute alcohol-associated pancreatitis can be reduced: a randomized controlled trial. Gastroenterology 2009;136:848-55.

14 Lappalainen-Lehto R, Koistinen N, Aalto M, et al. Goal-related outcome after acute alcohol-pancreatitis -- a two-year follow-up study. Addict Behav 2013;38:2805-9.

15 Tolstrup JS, Kristiansen L, Becker U, et al. Smoking and risk of acute and chronic pancreatitis among women and men: a populationbased cohort study. Arch Intern Med 2009;169:603-9.

16 Lindkvist B, Appelros S, Regnér S, et al. A prospective cohort study on risk of acute pancreatitis related to serum triglycerides, cholesterol and fasting glucose. Pancreatology 2012;12:317-24.

17 Sadr-Azodi O, Andrén-Sandberg Å, Orsini N, et al. Cigarette smoking, smoking cessation and acute pancreatitis: a prospective population-based study. Gut 2012;61:262-7.

18 Majumder S, Gierisch JM, Bastian LA. The association of smoking and acute pancreatitis: a systematic review and meta-analysis. Pancreas 2015;44:540-6.

19 Schuckit MA. Alcohol-use disorders. The Lancet 2009;373:492-501. doi:10.1016/S0140-6736(09)60009-X

20 Peris P, Parés A, Guañabens N, et al. Bone mass improves in alcoholics after 2 years of abstinence. J Bone Miner Res 1994;9:1607-12.
21 Aguilera MT, de la Sierra A, Coca A, et al. Effect of alcohol abstinence on blood pressure: assessment by 24-hour ambulatory blood pressure monitoring. Hypertension 1999;33:653-7.

22 Seitz HK, Bataller R, Cortez-Pinto H, et al. Alcoholic liver disease. Nat Rev Dis Primers 2018;4:16.

23 Jandíková $H$, Dušková M, Stárka L. The influence of smoking and cessation on the human reproductive hormonal balance. Physiol Res 2017;66:S323-31.

24 Taylor DH, Hasselblad V, Henley SJ, et al. Benefits of smoking cessation for longevity. Am J Public Health 2002;92:990-6.

25 Párniczky A, Kui B, Szentesi A, et al. Prospective, multicentre, nationwide clinical data from 600 cases of acute pancreatitis. PLoS One 2016;11:e0165309.

26 Szücs Ákos, Marjai T, Szentesi A, et al. Chronic pancreatitis: multicentre prospective data collection and analysis by the Hungarian pancreatic study group. PLoS One 2017;12:e0171420.

27 Weinberger AH, Platt J, Jiang B, et al. Cigarette smoking and risk of alcohol use relapse among adults in recovery from alcohol use disorders. Alcohol Clin Exp Res 2015;39:1989-96.

28 Yardley MM, Mirbaba MM, Ray LA. Pharmacological options for smoking cessation in heavy-drinking smokers. CNS Drugs 2015;29:833-45.

29 Mirbaba M. Heavy-Drinking smokers: pathophysiology and pharmacologic treatment options. Am J Psychiatry Resid J 2016;11:8-11.

30 Fucito LM, Hanrahan TH. Heavy-Drinking smokers' treatment needs and preferences: a mixed-methods study. J Subst Abuse Treat 2015;59:38-44.

31 McKelvey K, Thrul J, Ramo D. Impact of quitting smoking and smoking cessation treatment on substance use outcomes: an updated and narrative review. Addict Behav 2017;65:161-70.

32 Toll BA, Martino S, O'Malley SS, et al. A randomized trial for hazardous drinking and smoking cessation for callers to a quitline. $J$ Consult Clin Psychol 2015;83:445-54.

33 Crockett SD, Wani S, Gardner TB, et al. American Gastroenterological Association Institute Guideline on Initial Management of Acute Pancreatitis. Gastroenterology 2018;154:1096-101.

34 Working Group IAP/APA Acute Pancreatitis Guidelines. IAP/APA evidence-based guidelines for the management of acute pancreatitis. Pancreatology 2013;13:e1-15.

35 Samarasekera E, Mahammed S, Carlisle S, et al. Pancreatitis: summary of NICE guidance. BMJ 2018;362:k3443.

36 Isaji S, Takada T, Mayumi T, et al. Revised Japanese guidelines for the management of acute pancreatitis 2015: revised concepts and updated points. J Hepatobiliary Pancreat Sci 2015;22:433-45.

37 Chan A-W, Tetzlaff JM, Gøtzsche PC, et al. Spirit 2013 explanation and elaboration: guidance for protocols of clinical trials. BMJ 2013;346:e7586.

38 Banks PA, Bollen TL, Dervenis C, et al. Classification of acute pancreatitis--2012: revision of the Atlanta classification and definitions by international consensus. Gut 2013;62:102-11.

39 Yadav D. Reassessing the risk of pancreatitis with alcohol. Pancreas 2016;45:781-2.

40 Mosztbacher D, Hanák L, Farkas N, et al. Hypertriglyceridemiainduced acute pancreatitis: a prospective, multicenter, international cohort analysis of 716 acute pancreatitis cases. Pancreatology 2020;20:608-16.

41 Zádori N, Gede N, Antal J, et al. Early elimination of fatty acids in hypertriglyceridemia-induced acute pancreatitis (ELEFANT trial): protocol of an open-label, multicenter, adaptive randomized clinical trial. Pancreatology 2020;20:369-76.

42 Szentesi A, Párniczky A, Vincze Áron, et al. Multiple hits in acute pancreatitis: components of metabolic syndrome synergize each other's deteriorating effects. Front Physiol 2019;10:1202.

43 Hegyi $P$, Erőss $B$, Mátrai $P$, et al. The first definition for early chronic pancreatitis. Pancreatology 2018;18:S172.

44 Löhr JM, Dominguez-Munoz E, Rosendahl J, et al. United European gastroenterology evidence-based guidelines for the diagnosis and therapy of chronic pancreatitis (HaPanEU). United European Gastroenterol J 2017;5:153-99.

45 Platt L, Melendez-Torres GJ, O'Donnell A, et al. How effective are brief interventions in reducing alcohol consumption: do the setting, practitioner group and content matter? Findings from a systematic review and metaregression analysis. BMJ Open 2016;6:e011473.

46 WHO ASSIST Working Group. The alcohol, smoking and substance involvement screening test (assist): development, reliability and feasibility. Addiction 2002;97:1183-94.

47 Kaner EF, Beyer FR, Muirhead C, et al. Effectiveness of brief alcohol interventions in primary care populations. Cochrane Database Syst Rev 2018;2:CD004148. 
48 Kristenson $\mathrm{H}$, Öhlin $\mathrm{H}$, Hultén-Nosslin $\mathrm{MB}$, et al. Identification and intervention of heavy drinking in middle-aged men: results and follow-up of 24-60 months of long-term study with randomized controls. Alcohol Clin Exp Res 1983;7:203-9.

49 Pelli H, Lappalainen-Lehto R, Piironen A, et al. Risk factors for recurrent acute alcohol-associated pancreatitis: a prospective analysis. Scand J Gastroenterol 2008;43:614-21.

$50 \mathrm{Kim}$ S, Apelberg BJ, Avila-Tang E, et al. Utility and cutoff value of hair nicotine as a biomarker of long-term tobacco smoke exposure, compared to salivary cotinine. Int J Environ Res Public Health 2014;11:8368-82.

51 Andresen-Streichert H, Müller A, Glahn A, et al. Alcohol biomarkers in clinical and forensic contexts. Dtsch Arztebl Int 2018;115:309-15.

52 Mikó A, Erőss B, Sarlós P, et al. Observational longitudinal multicentre investigation of acute pancreatitis (GOULASH plus): follow-up of the GOULASH study, protocol. BMJ Open 2019;9:e025500.

53 Márta K, Szabó AN, Pécsi D, et al. High versus low energy administration in the early phase of acute pancreatitis (GOULASH trial): protocol of a multicentre randomised double-blind clinical trial. BMJ Open 2017;7:e015874.

54 Párniczky A, Mosztbacher D, Zsoldos F, et al. Analysis of pediatric pancreatitis (apple trial): Pre-Study protocol of a multinational prospective clinical trial. Digestion 2016;93:105-10.

55 Párniczky A, Abu-El-Haija M, Husain S, et al. EPC/HPSG evidencebased guidelines for the management of pediatric pancreatitis. Pancreatology 2018;18:146-60.

56 Hritz I, Czakó L, Dubravcsik Z, et al. [Acute pancreatitis. Evidencebased practice guidelines, prepared by the Hungarian Pancreatic Study Group]. Orv Hetil 2015;156:244-61.

57 Gholam PM. Prognosis and prognostic scoring models for alcoholic liver disease and acute alcoholic hepatitis. Clin Liver Dis 2016;20:491-7.

58 Hedman L, Katsaounou PA, Filippidis FT, et al. Receiving support to quit smoking and quit attempts among smokers with and without smoking related diseases: findings from the EUREST-PLUS ITC Europe surveys. Tob Induc Dis 2018;16:A14

59 Saunders JB, Aasland OG, Babor TF, et al. Development of the Alcohol Use Disorders Identification Test (AUDIT): WHO Collaborative Project on early detection of persons with harmful alcohol consumption--II. Addiction 1993;88:791-804.

60 Németh Z, Urbán R, Farkas J, et al. Hungarian adaptation of the long and the short form of the drinking motives questionnaire (DMQ-R). Magyar Pszichológiai Szemle 2012;67:673-94.

61 Kuntsche E, Kuntsche S. Development and validation of the drinking motive questionnaire revised short form (DMQ-R SF). J Clin Child Adolesc Psychol 2009;38:899-908.

62 Heatherton TF, Kozlowski LT, Frecker RC, et al. The Fagerström test for nicotine dependence: a revision of the Fagerström tolerance questionnaire. Br J Addict 1991;86:1119-27.

63 Capacities $\mathrm{HMoH}$ Az emberi erőforrások minisztériuma egészségügyi szakmai irányelve a dohányzásról való leszokás támogatásáról. Available: https://tudogyogyasz.hu2019

64 Varga L. Segítségnyújtás kockázatos ivók számára, 2006. Nationa addiction Institute

65 Petersen $\mathrm{OH}$, Sutton R. Ca2+ signalling and pancreatitis: effects of alcohol, bile and coffee. Trends Pharmacol Sci 2006;27:113-20.

66 Rencz F, Gulácsi L, Drummond M, et al. EQ-5D in central and eastern Europe: 2000-2015. Qual Life Res 2016;25:2693-710.

67 Lloyd A, Pickard AS. The EQ-5D and the EuroQol group. Value Health 2019;22:21-2.

68 Koncz B, Darvasi E, Erdősi D, et al. Lifestyle, prevention and risk of acute pancreatitis (lifespan): protocol of a multicentre and multinational observational case-control study. BMJ Open 2020;10:e029660.

69 Sahin-Tóth M, Hegyi P. Smoking and drinking synergize in pancreatitis: multiple hits on multiple targets. Gastroenterology 2017;153:1479-81. 